\title{
L’événementialité du phénomène selon Neue Phänomenologie in Frankreich
}

\author{
Yusuke IKEDA \\ Ritsumeikan University Kyoto
}

\section{Introduction}

Notre étude ${ }^{1}$ cherche à examiner la thèse fondamentale de Neue Phänomenologie in Frankreich ${ }^{2}$, selon laquelle la spécificité du mouvement phénoménologique récent en France consisterait à décrire le "phénomène phénoménologique » d'une nouvelle manière et précisément selon son «événementialité ». À cette fin, il ne suffit pas simplement de reconstruire ou de résumer cette thèse telle qu'elle est exposée dans l'ouvrage, mais il est également nécessaire de construire de manière progressive le concept général d’événement et celui de «phénomène phénoménologique » conçu selon son événementialité. D’après Tengelyi et Gondek, la tradition phénoménologique, dans son approche du phénomène en tant qu' «événementiel », demeure en effet toujours opératoire plutôt que thématique. ${ }^{3}$. Autrement dit, les auteurs ne cherchent pas à entreprendre une exposition panoramique du paysage de la phénoménologie française contemporaine, mais tentent avant tout de déceler le « concept opératoire » caché sans lequel ce mouvement philosophique ne serait pas possible.

Notre étude essaiera de répondre aux trois questions suivantes : (1) qu'est-ce que la «nouvelle phénoménologie en France »; plus précisément, dans quel contexte est-il possible de rendre compte de cette "nouvelle phénoménologie »? (2) Qu'entend-t-on couramment par " événement » ? Il faudra également esquisser très brièvement une préhistoire de la conception du phénomène en tant qu' « événementiel ». (3) Comment cette transformation du concept phénoménologique de phéno-

\footnotetext{
${ }^{1}$ Mes remerciements vont à Stéphane Finetti, Vincent Giraud, Roberto Formisano et Jean-Sébastien Hardy, sans l'aide desquels cette étude n'aurait pu paraître.

${ }^{2}$ Hans-Dieter Gondek et László Tengelyi, Neue Phänomenologie in Frankreich, Frankfurt a.M., Suhrkamp, 2011.

${ }^{3}$ Nous reprenons ici la fameuse distinction introduite par E. Fink entre « opératoire » et « thématique ». Voir Eugen Fink, « Operative Begriffe in Husserls Phänomenologie » (1957), dans Nähe und Distanz, Freiburg/München, Alber, 1976, p. 180-204.
} 
mène motive-t-elle une réorganisation remarquable de sa méthode, à laquelle Janicaud a reproché d'être un « tournant théologique » ?

\section{1/ Le point de départ : qu'est-ce que la « nouvelle phénoménologie en France " ?}

László Tengelyi et Hans-Dieter Gondek se proposent de déterminer et de clarifier en quoi consiste la nouveauté du mouvement philosophique récent en France. Amorcé silencieusement à partir des années 1960 et remarqué explicitement dans les années 1990, ce dernier prend comme source d’inspiration philosophique la phénoménologie husserlienne ainsi que la pensée tardive de Heidegger et, en même temps, prend de manière très remarquable ses distances des deux maîtres fribourgeois de la phénoménologie.

La formule même de "neue Phänomenologie in Frankreich » évoque d'emblée deux questions :

(1) En quel sens peut-on dire que ce mouvement phénoménologique est «nouveau »?

(2) L'expansion (actuelle ou potentielle) de ce mouvement phénoménologique se limite-t-elle à la France ? Dans quel contexte philosophique et spécifiquement « français » surgit cette nouvelle phénoménologie ?

Afin de répondre à ces questions, il faudra prêter attention — comme le font les auteurs — à des circonstances particulières : Dominique Janicaud est le premier à avoir attiré l'attention sur ce mouvement philosophique et sur sa " nouveauté », dans son livre polémique Le tournant théologique de la phénoménologie française publié en $1991^{1}$. Janicaud y soutient que ce mouvement phénoménologique (dont les représentants sont selon lui le dernier Merleau-Ponty, Emmanuel Levinas, Michel Henry, Jean-Luc Marion et Jean-Louis Chrétien) dépasse la limite méthodologique constitutive de la phénoménologie : la méthode de la fidélité aux phénomènes, qui interdit de bavarder sur ce qui ne se montre pas, sur ce qui n'apparaît pas, bref sur ce qu'on ne voit pas $^{2}$, par exemple Dieu ou «l'inapparent » (das Unscheinbare) chez le dernier Heidegger. Janicaud propose alors deux thèses d'après lesquelles (1) la " phénoménologie et [la] théologie font deux $»^{3}$, si bien que «la nouvelle phénoménologie en France » confond illégitimement l'une avec l'autre ; (2) la "phénoménologie de l'inapparent» (Phänomenologie des Unscheinbaren) de type heideggérien ne

\footnotetext{
${ }^{1}$ Dominique Janicaud, Le tournant théologique de la phénoménologie française, Combas, Éditions de l’Éclat, 1991, repris dans La phénoménologie dans tous ses états, Paris, Gallimard, 2009, p. 41149.

${ }^{2}$ C'est «l'athéisme méthodologique » qu'il postule dans son deuxième livre concernant cette polémique. Voir Dominique Janicaud, La phénoménologie éclatée, Combas, Éditions de l’Éclat, 1998, repris dans La phénoménologie dans tous ses états, op. cit., p. 151-276.

${ }^{3}$ Ibid., p. 149.
} 
conduit nulle part, et à celle-ci il faut opposer une "phénoménologie minimaliste »" La spécificité du mouvement philosophique que nous appelons, avec Tengelyi et Gondek, «la nouvelle phénoménologie en France » consiste ainsi pour Janicaud dans un dépassement "théologique », et donc philosophiquement inacceptable, de la limite de la méthode phénoménologique et dans un « avatar » douteux du concept phénoménologique de phénomène (consistant dans l'approche de «l'inapparent »).

Bien que nos auteurs ne souhaitent pas s'engager dans cette polémique ${ }^{2}$, le diagnostic de Janicaud détermine néanmoins leur point de départ et leur orientation d'ensemble, dans la mesure où ils se demandent si la thèse de Janicaud est encore tenable aujourd'hui ou s’il ne faut pas plutôt considérer ce mouvement phénoménologique d'une autre manière. Ainsi, il nous semble que la stratégie de nos auteurs consiste (1) à "mettre entre parenthèses » le diagnostic de Janicaud selon lequel phénoménologie et théologie seraient confondues dans le mouvement phénoménologique contemporain en France, et (2) à transformer le concept phénoménologique de phénomène, dont la légitimité est alors défendue par une réorganisation de la méthode phénoménologique elle-même. Nous ne pouvons plus parler dès lors comme Janicaud d'un dépassement et d'un «tournant théologique » du principe de la phénoménologie classique et d'un «avatar » illégitime de la phénoménalité du phénomène.

On peut ainsi déjà répondre aux deux questions formulées en introduction. (1) La «nouvelle phénoménologie en France » ne se caractérise pas comme «nouvelle » par rapport à la méthode « classique » de la phénoménologie parce qu’elle « dépasserait » (comme le soutient Janicaud) la limite méthodologique de la phénoménologie. Les auteurs de Neue Phänomenologie in Frankreich trouvent en effet la justification de leur thèse plutôt dans le fait que ce mouvement phénoménologique réorganise et révise profondément la méthode de « la phénoménologie classique ». Bref, on peut caractériser ce mouvement phénoménologique comme «nouveau » parce que sa méthode est tout à fait nouvelle et innovatrice par rapport à celle que pratiquaient les générations précédentes. (2) Par là, nous pouvons déjà conclure provisoirement que la «nouvelle phénoménologie » se situe dans un contexte spécifiquement français, parce que le débat sur « le tournant théologique » a eu lieu en France, mais aussi parce que la question même de savoir si le mouvement phénoménologique est aujourd'hui « éclaté » ou non ne se pose que si on s’intéresse à la situation

\footnotetext{
${ }^{1}$ Voir ibid., p. 263-273.

${ }^{2}$ Hans-Dieter Gondek et László Tengelyi, Neue Phänomenologie in Frankreich, op.cit., p. 20. Dans son dernier ouvrage, Tengelyi prend position de manière beaucoup plus explicite : "Allerdings kann er [Richir] unmöglich zu den » neuen Theologen « im Sinne von Janicaud gerechnet werden. Daraus folgt aber nur, dass die eigentliche Neuigkeit, die mit der Neuen Phänomenologie in Frankreich aufkommt, keineswegs in einer [theologischen] Wende besteht. Der polemische Eifer hat Janicaud in dieser Hinsicht bei all seinem Scharfsinn zur Prägung einer irreführenden Formel hingerissen » (László Tengelyi, Welt und Unendlichkeit, Freiburg/München, Alber, 2014, p. 289).
} 
typiquement française de la phénoménologie durant la dernière décennie du siècle dernier ${ }^{1}$.

Pour légitimer ces thèses, nos auteurs essaient d'élucider le concept de phénomène à partir de son caractère «événementiel », découvrant ce faisant l’intuition philosophique « opératoire » qui serait celle de la nouvelle phénoménologie française. Il ne s'agit pas ici de l'événement compris selon son acception physique ou même historique, mais de "l'événement du sens » (Sinnereignis) et de son mode de donation, donc de l'événementialité du phénomène phénoménologique, à savoir l'événement en tant qu'il advient au sujet sans être prévu, c'est-à-dire en tant qu'il excède la « conscience donatrice de sens » (sinngebendes Bewußtsein) ${ }^{2}$, ou encore en tant qu'il arrive au « sujet au datif $»^{3}$.

La conclusion de Neue Phänomenologie in Frankreich contient une remarque cruciale afin d'élucider l'orientation générale de la nouvelle phénoménologie française. Cette dernière aurait mis en évidence une "contradiction cachée » au sein de la phénoménologie husserlienne entre «l'idée d’une constitution transcendantale » d' « origine intellectualiste » et la « description des phénomènes » de " provenance empiriste ", contradiction qu'elle se serait ensuite efforcée de dissoudre. Comme l'expliquent en effet nos deux auteurs, «la donation de la donnée s'avère en tant qu'événement qui arrive (widerfährt) à la conscience et qui se manifeste de luimême » ${ }^{4}$. L'idée de constitution transcendantale est ainsi explicitement surmontée (et non pas « supprimée », comme c'est le cas dans la Phénoménologie de la perception de Merleau-Ponty) par la prise en compte du " caractère d'événement » (Ereignischarakter) du phénomène, qui reste toujours un excès pour la subjectivité transcendantale $^{5}$. De même, les notions quasi-empiristes de description et de donation sont également surmontées (et non pas supprimées, puisqu’il ne s’agit pas de revenir à la tradition métaphysique, purement spéculative, pré-kantienne) par la découverte

\footnotetext{
${ }^{1}$ La conclusion de Neue Phänomenologie in Frankreich résume brièvement les thèses « opératoires » (qui sont tacitement constitutives de la nouvelle phénoménologie française) en les opposant à celles de la "phénoménologie française » antérieure (voir Bernard Waldenfels, Phänomenologie in Frankreich, Frankfurt a.M., Suhrkamp, 1983). Ils prennent comme fil conducteur l'idée de phénoménologie exposée dans l'avant-propos de la Phénoménologie de la perception de MerleauPonty. Ils montrent que la différence entre cette dernière et la nouvelle phénoménologie française se cristallise dans leurs différentes approches (I) de la description analytique, (II) de la méthode de la corrélation intentionnelle, (III) de l'eidétique phénoménologique, (IV) de l'épochè et de la réduction, ainsi que (V) de l'idéalisme transcendantal de Husserl. Cf. Hans-Dieter Gondek et László Tengelyi, Neue Phänomenologie in Frankreich, op.cit., p. 666-671.

${ }^{2}$ Voir Edmund Husserl, Ideen zu einer reinen Phänomenologie und phänomenologischen Philosophie. Erstes Buch: Allgemeine Einführung in die reine Phänomenologie, dans Husserliana (désormais Hua), vol. III/1, Karl Schuhmann (éd.), Den Haag, Martinus Nijhoff, 1977, p. 120-121.

${ }^{3}$ Voir Hans-Dieter Gondek et László Tengelyi, Neue Phänomenologie in Frankreich, op.cit., p. 2034, et notamment, p. 23, 28 et p. 29-30.

${ }^{4}$ Ibid., p. 671.

${ }^{5}$ Ibid., p. 666-671.
} 
de l’événementialité des phénomènes, parce que la phénoménalité n’est plus alors conçue comme une donnée empirique qui ne se laisserait plus remettre en cause. Il s'agit en revanche dans la «nouvelle phénoménologie française » d'une analyse descriptive des phénomènes — in statu nascendi — à partir de leur événementialité. Ainsi, il est clair que l'orientation même de la nouvelle phénoménologie française consiste à opérer une synthèse de la " contradiction cachée » propre à la phénoménologie (husserlienne) et à élucider le phénomène phénoménologique (ou la phénoménalité) prise dans son événementialité. Mais que doit-on entendre plus précisément par « événement »; de quel type d' « événement » s’agit-il dans ce contexte ? Pouvons-nous attribuer en fin de compte à cette nouvelle conception de la phénoménalité un rôle philosophique aussi important ? Dans la section suivante, nous chercherons à expliciter la notion générale d'événement à travers trois étapes, à savoir une réflexion étymologique, ontologique puis phénoménologique, et ce afin de préparer le terrain pour répondre à notre question ultime.

\section{2/ Une topologie du concept d'événement}

Nous ne pouvons commencer directement par une phénoménologie de l'événement, puisque le concept d' « événement » est un concept plutôt vague qui en outre, nous semble-t-il, n’a pas été suffisamment élaboré dans la tradition philosophique. Il faudra ainsi tout d'abord étudier l'étymologie de notre concept-clé.

\section{A/ Une réflexion étymologique}

\section{L'étymologie minimale de la notion d'événement}

Événement est un "mot dérivé du latin evenire, d'après le précédent de avènement. Le substantif latin eventum, chose arrivée, est resté dans l'italien evento, anglais event ${ }^{1}$. Il est intéressant pour nous de le confronter avec son équivalent allemand : Ereignis. Sa forme verbale Ereignen (Eräugnen ou Ereugnen) ne renvoie en effet pas à evenire, mais plutôt (selon Jacob et Wilhelm Grimm) à contingere, accidere : Ereignen « est seulement réflexif et signifie uniquement manifester, se révéler $»^{2}$. Ainsi nous pourrions probablement insister sur la thèse selon laquelle le mode même d' " avènement » d'un événement n'est pas une simple manifestation ou apparition, mais s'accomplit dans un mode spécifique : il nous arrive, il advient sans que nous puissions prévoir son arrivée (si nous nous permettons de prendre au sérieux le fait que Ereignis équivaut à contingere et accidere). Ainsi Goethe écrit dans le Cho-

\footnotetext{
${ }^{1}$ Jean Auguste Scheler, Dictionnaire d'étymologie française; d'après les résultats de la science moderne, Bruxelles, Muquardt, 1888, p. 201.

2 «[Ereignen] steht nur reflexiv und bedeutet eigentlich erscheinen, sich offenbaren » (Jacob Grimm et Wilhelm Grimm, Deutsches Wörterbuch herausgegeben von der Deutschen Akademie der Wissenschaften zu Berlin, Leipzig, Hirzel, 1854-1971, t. III, p. 699).
} 
rus Mysticus de Faust: «Alles Vergängliche / ist nur ein Gleichnis ; / das Unzulängliche, / hier wird's Ereignis " ${ }^{1}$. Bref, l'événement est une chose arrivée ou un état advenu qui se manifeste (donc qui se montre à quelqu’un). L’événement est « la chose arrivée ", et cette « arrivée » implique le mouvement même de sa manifestation (erscheinen ou sich offenbaren) sans prévision possible. Car, nous le répétons, l'événement se manifeste dans le mode spécifique d'apparition que représentent contingere ou accidere.

\section{B/ Une réflexion ontologique sur l'événement en général}

\section{L'être des événements}

Sur la base de la détermination étymologique minimale du terme " événement », nous avons explicité le caractère essentiel de l'événement en tant que « chose arrivée ». On peut d’abord constater que «l'esse de l'événement est de prendre place, arriver ou se produire, mais pas d'exister $»^{2}$. Hacker nous donne des exemples tels que « la mort de César » qui n'existe pas, mais plutôt s'est passée au 44 av. J.-C., ainsi que l' « éruption du Vésuve » qui se passe ou non ce soir, alors que le Vésuve en tant que tel existe depuis longtemps ${ }^{3}$. Alors que, afin d'attester la proposition « le Vésuve existe ", nous n'avons pas besoin de vérifier l'arrivée même de son existence, il est nécessaire d'attester l'arrivée datable de l'éruption pour nier (ou affirmer, dans un futur indéterminé) la proposition «l'éruption du Vésuve est arrivée ce soir ». L'événementialité de l'événement n’est rien d'autre que le fait qu’il se passe et qu'il advient (parce que l'événement qui n’est pas arrivé, qui ne se manifeste pas, est, à proprement parler, selon l'étymologie un contre-sens) ${ }^{4}$, alors qu'on n'a pas besoin de vérifier l'arrivée d'une chose existante pour attester la proposition « elle existe ». Où arrive l'événement ? Nous pouvons y répondre en principe de deux manières, en soutenant qu'il arrive ou bien à notre monde spatio-temporel, ou bien à nous-mêmes.

\section{L'événement en soi et en général}

Si nous comprenons l'événement selon la première réponse, nous parlons d'un événement qui arrive à un système spatio-temporel. Si l'on interprète ce système

\footnotetext{
${ }^{1}$ Johann Wolfgang von Goethe, Faust, t. II, vers 12104 sq, dans Goethes Werke. Hamburger Ausgabe, E. Trunz (éd.), vol. III, Hamburg/München, Wegner, 1972, p. 364.

2 "The esse of events is to take place, happen or occur, but not to exist » (Peter M.S. Hacker, «Events, Ontology and Grammar», dans Events, R. Casti et A.C. Varzi (éd.), Brookfield/Singapore/Sydney, Aldershot, 1996, p. 81).

${ }^{3}$ Ibid.

${ }^{4}$ Mais nous pouvons quand même parler d'un « événement qui n’arrive pas », si nous entendons par là, par exemple, des événements arrivés dans un roman ou un récit. La fiction a habituellement - son propre système spatio-temporel, ou bien son propre «monde », qui n'est pas tout à fait notre monde. Nous ne thématiserons pas ici cette co-implication des « mondes ", à savoir celle entre la réalité et la fiction.
} 
comme celui de la physique, il s'agit là d’un événement physique qui est déterminable selon les lois physiques. Nous pouvons comprendre par «monde spatiotemporel » également un monde dans lequel notre histoire se déploie. L’événement doit dans ces deux cas arriver à un système (physique, historique, etc.) pour qu'il puisse arriver. Ce «système » ou "monde " n'est autre que l'espace ou le cadre ontologiquement normatif sans lequel nul événement ne peut arriver. Par exemple : nul physicien n’écrit un article scientifique sur un événement qui ne peut en principe jamais s'insérer dans un cadre spatio-temporel scientifiquement accessible, parce que ce dernier ne pourrait jamais être reconnu comme événement physique (c’est pourquoi le «chat de Schrödinger » constitue un problème pour les physiciens). Pour reconnaître une chose en tant qu’événement, il faut être engagé dans un certain « système ", « cadre » ou « monde » normatif.

\section{L'événement par rapport à nous}

Le fait qu'un événement arrive à un système physique ou historique ne semble pas être une condition suffisante pour le connaître en tant qu'événement, si nous entendons par «connaître quelque chose en tant qu'événement» le fait même de parler d'un événement qui est réellement arrivé. Autrement dit, il pourrait y avoir une multiplicité indéfinie d'événements - ceux qui sont arrivés à notre monde auxquels nous n'avons pas un accès tel qu'il nous permette de les reconnaître en tant que tels. Si nous voulons parler d'un événement en tant qu'événement vrai ou réel, il faut qu'il puisse nous advenir. Ce «rapport à nous » de l'événement a un pouvoir épistémologiquement normatif. Nous pourrions caractériser cette thèse, faute de mieux, comme celle de la normativité de l'évaluation sans laquelle (par exemple pour Nietzsche) personne ne saurait vivre ${ }^{1}$ : s'il n'y avait pas d'évaluation d'un événement par rapport aux autres, personne n’aurait accès à l'événement qui se laisse distinguer des autres événements. Afin de parler d'un événement en tant qu’événement, il nous semble nécessaire de le considérer par rapport à d'autres événements.

\section{C/ Une réflexion phénoménologique}

Le sujet ne prend pas toujours l'initiative de l'évaluation

En prenant au sérieux ce pouvoir épistémologiquement normatif (ou pouvoir d'évaluation), nous avons sans doute tendance en tant que philosophes à tirer une

\footnotetext{
${ }^{1}$ Il ne s'agit sans aucun doute pas chez Nietzsche de quelque norme ou normativité éternellement vraie et fixée qui serait constitutive de la vie en la totalisant. «Daß eine Menge Glauben da sein muß, daß geurtheilt werden darf, daß der Zweifel in Hinsicht auf alle wesentlichen Werthe fehlt:das ist Voraussetzung alles Lebendigen und seines Lebens. Also daß etwas für wahr gehalten werden muß, ist nothwendig; nicht, daß etwas wahr ist » (Nietzsche Werke. Kritische Gesamtausgabe, Sect. VIII, vol. II : Nachgelassene Fragmente, Herbst 1887 bis März 1888, édité par G. Colli et M. Montinari, Berlin/New York, de Gruyter, 1970, p. 16).
} 
conclusion différente, subjectiviste : si l'on croit qu'il doit y avoir une subjectivité humaine qui pose la norme, qui évalue la valeur d'un événement, si l'on croit en l'existence nécessaire d'un sujet qui ne subit pas simplement la norme, mais plutôt l'établit, on a tendance à soutenir la thèse subjectiviste selon laquelle le sujet, qui est l'agent ou le fondateur de cette normativité (ou de cette évaluation), constitue la condition nécessaire (transcendantale ou métaphysique) du pouvoir normatif de l'évaluation en général. Mais il nous semble que nul phénomène n’atteste cette thèse dans sa généralité. Nous subissons en effet beaucoup de normes (d'évaluations) dont le fondateur est tout à fait inconnu. On reconnaît que la première guerre mondiale est un événement historique décisif, non pas parce qu'un historien a formulé ce jugement, mais plutôt parce qu'il est difficile faire sens de l'histoire du $X X^{e}$ siècle sans considérer cet événement. Nous pouvons alors formuler une thèse inverse à la thèse subjectiviste: un événement (dans notre exemple il s'agit d'un événement historique) est d'autant plus important qu'il ne peut pas nous échapper. Cette thèse n’est pas pour autant «a-subjectiviste », parce qu'un sujet y est bien présent, mais « au datif », alors que la thèse subjectiviste conçoit le sujet en tant qu'agent de l'évaluation (au nominatif).

Une réduction phénoménologique de l'événement à son mode d'expérience L'expérience ordinaire et l'expérience extra-ordinaire

Nous accomplissons ci-dessous une réduction phénoménologique, à savoir une reconduction de l'événement à deux modes de «l'expérience au datif ». Il faut constater qu'il y a des événements dont l' «arrivée » ou l' " avènement » au sujet se laisse comprendre comme simple exemplification d'une règle ou d'une loi. Un vol de porte-monnaie dans le métro de Paris n'est qu'un cas banal, quotidien et normal selon la statistique criminelle, dont la particularité ne peut se laisser saisir en consultant les dossiers de la police nationale ${ }^{1}$. D’une part, cet événement n’est bien sûr pas prévu par le propriétaire du porte-monnaie volé (ainsi il est «chose arrivée sans prévision »), mais d'autre part, il n’a pas le pouvoir de changer la loi ou la règle statistique en tant que telle. Son arrivée confirme ou infirme l'expérience ordinaire. Dans ce cas, le sujet auquel l'événement arrive est le sujet de l'expérience ordinaire : il est sans doute le citoyen familier à cet ordre normal des choses. En revanche, nous entendons normalement par « événement historique » ce qui arrive d'une façon telle qu'il change profondément (donc «a-normalise ») l'ordre social, le paysage de l'histoire et notre vie. C'est pourquoi la Réforme protestante est bel et bien un « grand événement historique » : elle n’est pas l'exemplification ou la con-

\footnotetext{
${ }^{1}$ Dans ce sens-ci, on pourrait dire avec Waldenfels qu'il est un des « Ereignissen ohne Eigenschaften, die streng genommen niemandem widerfahren » (Bernard Waldenfels, "Die Macht der Ereignisse », dans Ereignis auf Französisch. Vom Bergson bis Deleuze, M. Rölli (éd.), München, Fink, 2004, p. 448).
} 
séquence d'un ordre religieux, social et politique, mais ce qui l'excède et le change en profondeur. Son arrivée est ainsi une expérience extra-ordinaire : celui à qui l'événement historique arrive n'est en effet rien d'autre que le sujet d'une expérience extra-ordinaire. Mais celui à qui il arrive n'est sans doute dans la plupart des cas que le témoin de cet événement, plutôt que le sujet de ce dernier. De toute évidence, le témoin n'est pas nécessairement l'agent de cet événement, mais on ne peut pas dire non plus qu'il le subit de manière exclusivement passive. Il fait plutôt expérience Erfahrung mit etwas machen - «avec » ou à l'occasion de cet événement et ainsi avec lui-même. Il « fait expérience avec » l'ouverture ou l'accès même à un nouvel ordre dont la possibilisation n'est rien d'autre que le fait que cet événement lui est effectivement arrivé ${ }^{1}$. Ces deux modes d' « expérience au datif » correspondent à la «polarisation » de l'expérience :

Die starke Variante [der Erfahrung] besagt, daß wir Erfahrungen machen und durchmachen, die uns und unsere Welt verändern, während die schwache Variante sich auf das Vorhandensein oder Nichtvorhandensein solcher Erfahrungsdaten beschränkt, die unsere Vorannehmen und Vorentwürfe bestätigen oder entkräften. Hinzu kommt eine Polarisierung der Erfahrung, die sich zwischen Gewöhnung und Überraschung bewegt ${ }^{2}$.

\section{L'expérience sans précédent (beispiellos) en tant qu'expérience originaire}

Nous avons dégagé deux catégories constitutives fondamentales de l'expérience, à savoir l'« accoutumance » (Gewöhnung) et la « surprise » (Überraschung). Une expérience surprenante ou étonnante peut arriver à quelqu'un seulement s'il n'a pas anticipé son arrivée. En ce sens, elle n'est jamais le cas particulier d'une règle générale : si nous voulons saisir ce qui fait l'expérience de la surprise en tant que telle, nous ne pouvons opérer avec le schème de l'exemplification des règles générales.

\footnotetext{
${ }^{1}$ Si une personne n'avait pas accès au nouvel ordre en tant qu'effet d'un événement, malgré son arrivée « réelle » ou «objective », elle ne pourrait être témoin de cet événement au sens strict, parce qu'elle ne pourrait saisir ce qui s'est passé. Pour elle, ce serait une expérience chaotique ou absurde dans laquelle elle souffrirait tout à fait sans raison. Imaginons que la Maréchale, protagoniste de Der Rosenkavalier de Richard Strauss et Hugo von Hofmannstahl, ne laisse pas son jeune amant Octavian la quitter. Elle n'aurait alors rien compris à ce qu'il s'est passé entre lui et Sophie, sa future femme, et elle ne verrait pas que ce fait a changé totalement son ancienne relation avec Octavien. On pourrait dire en ce sens que le nouvel amour entre Sophie et Octavian " a-normalise » l'ancien amour de la Maréchale. Un nouvel ordre s'établit. Mais l'ancien amour était autrefois normalisé : la discussion du matin dans le lit n'était qu'un cas normal avant l'entrée de Sophie. Dans ce cas de figure, la Maréchale ne jouerait plus le rôle de témoin de la fin de son amour qu'elle joue dans l’opéra que nous connaissons.

${ }^{2}$ Bernard Waldenfels, Bruchlinien der Erfahrung. Phänomenologie, Psychoanalyse, Phänomenotechnik, Frankfurt a.M., Suhrkamp, 2002, p. 30. La discussion dans le lit entre la Maréchale et Octavian se passe sur le mode de l'expérience accoutumée. Le fait qu'Octavian tombe amoureux de Sophie, en revanche, arrive à la Maréchale plus ou moins sur le mode de la surprise. Ce qui se passe dans la dernière scène de l'opéra est une re-normalisation du nouvel ordre. Autrement dit, la Maréchale a accepté le nouvel amour et a dit adieu à ses beaux jours pour re-normaliser une fois pour toutes la nouvelle situation qui leur est arrivée.
} 
Autrement dit, l'expérience surprenante se caractérise comme une "expérience sans précédent » (beispiellos) ${ }^{1}$. L'invention de la forme de la sonate dans l'histoire de la musique classique est bel et bien un événement singulier qui n'a pas de précédent : elle engendre en revanche beaucoup d'exemples par sa forme même, tels que la sonate en mi bémol majeur pour piano ou la symphonie ${ }^{\circ} 100$ en sol majeur de Joseph Haydn, « Hammerklavier » ou « Eroica » de Ludwig van Beethoven, la sonate en si mineur de Franz Liszt, la huitième de Gustav Mahler, la quatrième symphonie de Dmitri Chostakovitch ${ }^{2}$, etc. La singularité des événements surprenants et, corrélativement, l'expérience surprenante résident dans le pouvoir de rendre possibles des exemples. C'est ainsi un pouvoir originaire ${ }^{3}$. Cette possibilisation originaire ouvre la dimension dans laquelle l'exemplification devient possible. La révolution française ne se laisse pas comprendre comme un exemple d'autre chose qu'ellemême, mais rend possible la vie du citoyen moderne ou le nationalisme moderne dont on peut trouver aujourd'hui partout dans le monde de bons et mauvais exemples : la révolution française n'est pas un exemple de la vie du citoyen moderne ou de nationalisme moderne, mais est ce qui les a rendus possibles. Elle est donc en tant que telle un événement singulier et originaire. Par contre, on trouve couramment des expériences qui sont l'exemplification de quelque règle générale. Celles-ci renvoient à l'expérience banale ou normalisée. Il est évident que l'événement physique particulier doit, en tant qu'objet de la physique, toujours être compris comme exemple d'une règle physique qui revendique sa généralité et non pas une singularité, parce que la physique ne s'intéresse au cas extraordinaire ou au contre-exemple que s’il désavoue la généralité de la théorie physique. La tâche infinie de la physique n'est autre que celle de remplacer une vieille théorie par une autre capable d'intégrer les contre-exemples de la précédente, donc de re-normaliser la théorie physique. Il est clair que l'expérience sans précédent ne coïncide pas pour sa part dans le contexte de l'histoire de la philosophie avec l'empirisme classique ${ }^{4}$.

\footnotetext{
${ }^{1}$ Voir Bernard Waldenfels, Hyperphänomene. Modi hyperbolischer Erfahrung, Frankfurt a.M., Suhrkamp, 2012, p. 189-197.

${ }^{2}$ Il faut faire attention ici : tous ces exemples de sonate ont à leur tour changé ou bouleversé (et ils changent et bouleversent toujours) radicalement notre représentation de la forme sonate. Ainsi, ils l'a-normalisent et la re-normalisent (a-normalisation re-normalisante ou re-normalisation anormalisante). Faute de place, nous ne pourrons pas analyser cette co-implication dans notre étude.

${ }^{3}$ Pour préciser la notion d' « origine » (Ursprung) ou d' « originaire » (Ursprüngliches), voir Walter Benjamin, Ursprung des deutschen Trauerspiels, dans Gesammelte Schriften, R. Tiedemann (éd.), vol. I/1, Frankfurt a.M., Suhrkamp, 1978, p. 226. Günter Figal explique la belle expression de Benjamin de la manière suivante : «Das Ursprüngliche ist wie der Strudel im Fluß. Es erklärt sich aus keinem Zusammenhang, sondern stiftet einen Zusammenhang erst; es gehört in keine Entwicklung, sondern was zu ihm als Sichentwickelndes gehört, wird erst vom Ursprünglichen her als solches erkannt » (Günter Figal, Gegenständlichkeit, Tübingen, Mohr Siebeck, 2006, p. 31).

${ }^{4}$ C'est pourquoi la philosophie non-empiriste de l'expérience n'est jamais un contre-sens. Voir Bernard Waldenfels, « Die Macht der Ereignisse », dans Ereignis auf Französisch, op.cit., p. 447.
} 
Le phénomène en tant qu'événement dans la tradition phénoménologique

Cette approche phénoménologique du phénomène (l'événementiel qui renvoie à son expérience singulière et originaire, ainsi qu'à son pouvoir de possibilisation originaire) s’était probablement déjà amorcée dans les années 1920 et 1930 chez Martin Heidegger et chez Eugen Fink, atteignant chez les deux philosophes de Fribourg un point culminant après la deuxième guerre mondiale. Ces derniers considèrent en effet la réflexion sur la phénoménalité du phénomène à partir de son originalité comme une des tâches indispensables (ou même comme la tâche) de la phénoménologie. Bien que nous ne puissions détailler leur démarche respective (ce serait sans doute un grand sujet de recherche systématique), on peut indiquer leur différence de la manière suivante. Heidegger tente d'élucider de manière grandiose le «Seyn» - phénomène par excellence de son entreprise philosophique (ou «Denken ») — par la «Seinsgeschichte » : il essaye de comprendre l'être même de l'étant en le reconduisant à son origine historique en tant qu'événement qui est nécessairement « celé » (verborgen) ${ }^{1}$. Fink tente en revanche de formuler sans cesse la thèse selon laquelle, premièrement, le phénomène doit être élucidé non pas à partir d'un phénomène intramondain, mais à partir du phénomène d'origine des phénomènes intramondains, à savoir «le monde »; deuxièmement, Fink comprend le monde comme l'événement par excellence :

Die Welt "wird”: das heißt nicht, ein Ding entsteht oder ein Ereignis läuft ab, es wird der Spielraum für Dinge und Ereignisse — es entsteht das Entstehenkönnen und Vergehenkönnen von Seiendem, - es ereignet sich das Sichereignenkönnen von Ereignissen. Es “geschieht” der Raum, die Zeit, das Erscheinen².

Nous pouvons résumer et articuler cette pensée finkienne de la manière suivante : (1) Fink entend par "monde » l'arrivée même du monde, ainsi il s'agit là (2) du monde au sein duquel tout événement arrive, le monde dont le pli se déploie comme " espace, temps, apparaître ». (3) Nous pouvons dire que le monde est ici interprété à partir de sa capacité de rendre possible cette arrivée même, c'est-à-dire à partir de son pouvoir d'encadrement de tout événement ${ }^{3}$. Nous pouvons ajouter que (4) Fink développe sa conception du phénomène du monde en tant qu'événementiel en prenant comme modèle ce que nous pouvons appeler «l'événement silencieux » ${ }^{4}$, car il

\footnotetext{
${ }^{1}$ Voir Rudolf Wansing, «Im Denken erfahren. Ereignis und Geschichte bei Heidegger », dans Ereignis auf Französisch, op. cit., p. 81-102.

${ }^{2}$ Eugen Fink, Welt und Endlichkeit, F.A. Schwarz (éd.), Würzburg, Königshausen \& Neumann, 1990, p. 205.

${ }^{3}$ Au sujet de cet « encadrement », voir notre étude, « Transzendentaler Schein und phänomenologische Ursprünglichkeit — Welterfahrung bei Husserl und Fink », dans Horizon, numéro 3/1, 2014, p. 94.

${ }^{4}$ Nous entendons par « événement silencieux » un événement qui arrive silencieusement, bien qu'il transforme ou a-normalise profondément et originairement un ordre ou notre vie elle-même : un événement se produit de façon silencieuse s'il nous échappe d'autant plus que nous pouvons difficilement suivre la longue série de ses effets et de ses causes (autrement dit, l'événement nous
} 
parle de «l'oubli du monde » (Weltvergessenheit) ${ }^{1}$. Le monde a été en effet oublié dans la philosophie, parce qu'il arrive — «die Welt ereignet sich » — très ou trop silencieusement pour l'optique de la philosophie. C'est pourquoi la philosophie est tombée selon Fink dans «l'oubli du monde ». Nous concluons que (6) le monde en tant qu'événementiel est en ce sens sans aucun doute un phénomène absolument singulier, parce qu'il ne peut jamais avoir quelque chose d'autre que lui-même ou

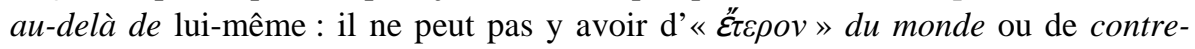
monde. Pour comprendre sa singularité, il suffit d'évoquer une thèse très célèbre du Tractatus logico-philosophicus de Wittgenstein, que Fink pourrait (du moins dans ce contexte) s'approprier : "Die Welt ist alles, was der Fall ist » ${ }^{2}$.

Parce que Heidegger et Fink tentent par une entreprise grandiose non seulement d’interpréter la phénoménalité même du phénomène (ou l'être même de l'étant) à partir de son originalité proprement événementielle, mais aussi de réviser la grande tradition métaphysique et ses catégories à partir de cette dernière, les deux différentes approches de l'événement que nous avons très brièvement esquissées impliquent des critiques différentes de la métaphysique : alors que Heidegger révise fortement le principium rationis (der Satz vom Grund) et la catégorie métaphysique de la cause (Ursache) $)^{3}$, le projet finkien consiste à réfléchir sur la catégorie du tout (All) ou de totalité (Allheit), qui, dans la mesure où elle se réfère au monde, reste un concept opératoire négligé de la métaphysique ${ }^{4}$. Ainsi l'entreprise heideggérienne se laisse comprendre non seulement comme une critique de l'onto-théologie (selon une terminologie issue de Kant), mais aussi comme une lutte contre la tradition onto-

échappe, si la série de ses effets et de ses causes nous échappe, ou l'événement est silencieux, si la série de ceux-ci est silencieuse). Ce type d'événement doit bel et bien être un objet pour un historien perspicace, si nous appliquons cette catégorie dans le domaine de l'historiographie. L'événement silencieux est une sorte de vague silencieusement expansive sans laquelle la mer ne serait pas ce qu'elle est.

${ }^{1}$ Voir Eugen Fink, Welt und Endlichkeit, op. cit., p. 205 sq. L'expression « Weltvergessenheit » est introduite dans le dernier ouvrage de László Tengelyi dans un sens presque identique à son usage finkien. Tengelyi ne renvoie pourtant pas à Fink, mais achève plutôt le motif que Fink a développé en suivant une autre voie. Voir László Tengelyi, Welt und Unendlichkeit, op. cit., p. 303-305. Comme Fink, Tengelyi considère la philosophie critique de Kant comme l'événement même de la tradition philosophique decet « oubli ».

${ }^{2}$ Ludwig Wittgenstein, Tractatus Logico-philosophicus, thèse I, dans Werksausgabe, vol. I, Frankfurt a.M., Suhrkamp, 1989, p. 11.

${ }^{3}$ Voir Martin Heidegger, Der Satz vom Grund, dans Heideggers Gesamtausgabe, vol. X, P. Jaeger (éd.), Frankfurt a.M., Klostermann, 1997.

${ }^{4}$ Voir Eugen Fink, Welt und Endlichkeit, op. cit., et Eugen Fink, Alles und Nichts. Ein Umweg zur Philosophie, Den Haag, Martinus Nijhoff, 1959. C'est pourquoi le "monde » devient, dit Kant, " "crux" pour le philosophe " (voir Immanuel Kant, De mundi sensibilis atque intelligibilis forma et princiis (1770), dans Immanuel Kant Werksaugabe, vol. V/1: Schriften zur Metaphysik und Logik, W. Weischedel (éd.), Frankfurt a.M., Suhrkamp, 1977, p. 26). Nous traduisons. 
protologique de la métaphysique ${ }^{1}$, alors que Fink opère une réhabilitation très originaire de la tradition cosmo-hénologique ${ }^{2}$.

\section{3/ La nouvelle phénoménologie française et l'événementialité du phénomène phénoménologique}

Dans cette dernière section de notre étude, nous allons mettre à l'épreuve la thèse même de Neue Phänomenologie in Frankreich à partir des résultats de notre réflexion sur la notion d'événement. À cette fin, nous ne pourrons examiner que deux figures de la «nouvelle phénoménologie française », soit celles de Jean-Luc Marion et de Marc Richir. Notre choix n’est pas arbitraire : Tengelyi et Gondek considèrent en effet leurs positions philosophiques respectives comme les « deux pôles extrêmes d'un champ commun » ${ }^{3}$. Ce "champ commun » relève de la tâche ou de l'orientation de la "nouvelle phénoménologie française », que nous avons étudiée dans la première section de ce travail : la tâche d'une synthèse de la « contradiction cachée » propre à la phénoménologie. Nous étudierons ainsi deux formes de la « nouvelle phénoménologie », (a) en présentant sa « polarisation » comme une réorganisation du système d’une phénoménologie qui réfléchit sur l'événementialité du phénomène et (b) en évaluant les résultats auxquels Marion et Richir parviennent sur la base de leurs contributions à la tradition métaphysique, de telle sorte que se révèlent la «proximité et la distance » de ces deux nouveaux phénoménologues français avec la tradition phénoménologique de Fribourg.

\footnotetext{
${ }^{1}$ Il s'agit d'un néologisme que nous avons forgé à partir de l'expression «katholou-protologie », qui est déjà plus ou moins acceptée dans les recherches francophones sur la métaphysique d'Aristote : voir R. Brague, Aristote et la question du monde, Paris, PUF, 1988, p. 110 et p. 415514, et. Olivier Boulnois, « Heidegger, l’onto-théo-logie et les structures médiévales de la métaphysique ", in Quaestio. Annuario di storia della metafisica, numéro 1, 2001, p. 379-406. Voir aussi Jean-François Courtine, Les catégories de l'être. Étude de philosophe ancienne et médiévale, Paris, PUF (coll. «Épiméthée »), 2003, p. 192.

${ }^{2}$ Il s'agit d'un autre néologisme reposant cette fois sur le terme d' " hénologie ». On peut trouver des prémisses implicites de ce que nous appelons ici provisoirement "cosmo-hénologie » dans la philosophie «méontique » que Fink développe dans ses manuscrits inédits. Voir Ronald Bruzina, " Hinter der ausgeschriebenen Finkschen Meditation: Meontik-Pädagogik », in Eugen Fink Sozialphilosophie Anthropologie Kosmologie Pädagogik Methodik, A. Böhmer (éd.), Würzburg, Königshausen und Neumann, 2006, p. 193-219.

${ }^{3}$ Hans-Dieter Gondek et László Tengelyi, Neue Phänomenologie in Frankreich, op.cit., p. 28, mais voir aussi p. 25-26, p. 228 et p. 259. Nous traduisons.
} 


\section{A/ La nouvelle phénoménologie française n’entreprend pas une spécification descriptive des événements, mais une transformation du concept du phénomène}

Marion et la phénoménologie de la donation en tant que réflexion sur la phénoménalité du phénomène

La contribution de Marion à la phénoménologie se présente comme une " phénoménologie de la donation ». Nous n'entrerons pas ici dans la question directrice qui anime son projet, parce qu'il faut admettre qu'elle pourrait résider sans doute dans une réhabilitation et même dans une continuation de certains aspects de la tradition " théologique " ${ }^{1}$. Mais cela ne signifie pas que la philosophie de Marion ne se laisse élucider qu'à partir de son intérêt théologique. Autrement dit, nous soutenons (selon la stratégie même de Neue Phänomenologie in Frankreich) que les résultats de sa recherche phénoménologique ne relèvent pas entièrement d'une réflexion théologique. Pour éviter l'enchevêtrement de motifs qui parurent à Janicaud exclusivement " théologiques ", nous expliciterons les résultats philosophiques de la phénoménologie de Marion de la manière suivante.

Marion thématise et développe le concept phénoménologique de la « donation » (Gabe, Gegebenheit ou Gebung) dans un contexte originairement non-théologique (à savoir une interrogation sur le statut de l'Anschauung et de l'intention ou de la signification dans la théorie de l'intentionnalité des Logische Untersuchungen) ${ }^{2}$ en parvenant ainsi à une réflexion sur la phénoménalité du phénomène phénoménologique. Il s’agit en effet pour Marion de radicaliser la phénoménologie et sa méthode, c’est-à-dire la réduction. Le point de départ de sa " phénoménologie de la donation » est bien exemplifié par la citation suivante :

La phénoménologie commence en 1900-1901 parce que, pour la première fois, la pensée voit apparaître l'apparaissant dans l'apparition ; elle n’y parvient qu'en concevant l'apparaître lui-même non plus comme un "donné de conscience", mais bien comme la donation à la conscience (voire par la conscience) de la chose même, donnée sous le mode de l'apparaître et dans toutes les dimensions de celui-ci (intuition, intention, et leurs variations) [...]. La percée (Durchbruch) phénoménologique ne consiste ni dans l'élargissement de l'intuition, ni dans l'autonomie de la signification, mais dans la primauté seule inconditionnée de la donation du phénomène ${ }^{3}$.

Pour justifier et articuler cette thèse, Marion fait un grand détour en distinguant « trois étapes » dans l’histoire de la méthode de la réduction, qui correspondent à

\footnotetext{
${ }^{1}$ Ses analyses sont explicitement déterminées par le motif théologique dans les ouvrages suivants : Jean-Luc Marion, L'idole et la distance, Paris, Grasset (coll. « Figures »), 1977 ; Dieu sans l'être, Paris, PUF (coll. « Quadrige »), [1982] 2010.

${ }^{2}$ Hans-Dieter Gondek et László Tengelyi, Neue Phänomenologie in Frankreich, op.cit., p. 154155. Voir Jean-Luc Marion, Réduction et donation, Paris, PUF (coll. «Épiméthée »), 1989, p. 1163.

${ }^{3}$ Jean-Luc Marion, Réduction et donation, op. cit., p. 53.
} 
trois types de donation (Marion entend en réalité ces « trois étapes » comme une radicalisation progressive de la phénoménologie $)^{1}$ : la réduction transcendantale à l'objectité (Husserl), la réduction ontologique à l'être (Heidegger) et « la réduction de toute revendication à la forme pure de l'appel $»^{2}$ (Marion). Bien que Marion parle de « la forme pure », il ne s'agit ni d'une «formalisation » de l'appel qui a lieu dans la vie quotidienne (l’appel dans la rue, l'appel au téléphone, etc.), ni de l’Anspruch des Seins qui a lieu selon lui dans « l'ennui des profondeurs " ${ }^{3}$. La «troisième percée » de Marion consiste plutôt dans une radicalisation critique de la pensée heideggérienne, car l'instance de l'appel ne doit pas nécessairement être identifiée selon lui avec l' « être ». Bien que Marion critique fortement dans ce contexte la pensée tardive de Heidegger, c’est elle en tout cas qui constitue son point de départ : «Que la revendication puisse s'exercer en un autre nom que celui de l'être, au nom d'un autre que l'être, Heidegger l'a d'ailleurs incidemment avoué, en opposant à l'Anspruch des Seins l'Anspruch des Vaters » ${ }^{4}$. Ici, il faut faire attention : une "réduction à la forme pure de l'appel » ne signifie sans doute jamais une reconduction absorbante de l'Anspruch des Seins à l'Anspruch des Vaters (celle-ci serait une réduction entièrement théologique), mais son enjeu consiste plutôt dans la mise en suspens de la fixation de l'instance ou de l'agent de l'appel. Cette «mise en suspens » de la " troisième réduction » qui évoque évidemment des questions assez légitimes (par exemple, qui peut répondre à un appel dont l'agent est complètement inconnu ou indéterminé ?), rend manifeste selon Marion l' «a priori » même de l'appel et de la revendication :

L'indétermination de l'instance revendicatrice rend seule possible la revendication, qui, sinon, ne surprendrait pas et donc ne provoquerait nul interloqué. Cet $a$ priori s'exerce d'autant mieux qu'il ne s'identifie pas, puisqu'il ne consiste qu'en sa pure reconnaissance par l'interloqué. La revendication elle-même s'atteste dans la reconnaissance, par l'interloqué, qu'il encourt une revendication ${ }^{5}$.

Il nous faut articuler les implications de cette pensée. Marion comprend l'appel à partir de son caractère "interloqué ». Il doit donc y avoir quelque événement où l'appel interloque ou surprend quelqu'un pour que l'appel puisse se révéler en tant qu'appel. Si nous ne sommes pas du tout étonnés, interloqués ou stupéfaits par un appel, nous ne reconnaissons en effet pas cet appel en tant qu'appel dans son originalité. De toute évidence, Marion comprend l'appel à partir de ce que nous nommons "l'expérience extra-ordinaire » et "l'expérience originaire » qui rend possible sa forme unique. L' «a priori » de l'appel que Marion a bien remarqué n'est dès

\footnotetext{
${ }^{1}$ C'est la thèse même de Réduction et donation. Voir Hans-Dieter Gondek et László Tengelyi, Neue Phänomenologie in Frankreich, op.cit., p. 156-159.

${ }^{2}$ Jean-Luc Marion, Réduction et donation, op. cit., p. 296.

${ }^{3}$ Voir ibid, p. 280-289.

${ }^{4}$ Ibid. p. 294.

${ }^{5}$ Ibid. p. 302.
} 
lors rien autre que l' « a priori » de la reconnaissance de l'appel en tant qu'appel ${ }^{1}$ : un a priori qui consiste ainsi, nous semble-t-il, dans ce que nous appelons pouvoir normatif. Mutatis mutandis: Marion parle d'une donation très spécifique, c'est-àdire de la donation sans donateur ${ }^{2}$ (ou plus précisément, la donation sans identification du donateur).

Cela veut dire qu'une phénoménologie de la donation élucide la donation (ou l'appel) à partir de son « avènement » à quelqu'un, mais aussi à partir de son pouvoir normatif, parce que cet avènement figure originairement l'espace (ou l' «a priori ») même de la donation sans lequel on ne peut la reconnaître en tant que donation. Ainsi nous nous permettons de conclure que ces deux thèses ne sont possibles que si l'on entend la donation ou l'appel à partir de son événementialité telle que nous l'avons caractérisée dans la section précédente. S’il est vrai que l’on peut déterminer, comme Marion le croit, ces «trois étapes de la réduction » comme la voie radicale propre au mouvement phénoménologique qui conduit vers une phénoménologie de la donation, il s'agit là en réalité d'une transformation tacite du concept du phénomène phénoménologique, à savoir du phénomène en tant qu'événement originaire. C'est pourquoi il n'est pas question d'une simple spécification descriptive des événements dans la phénoménologie de la donation de Marion :

Tenir cette radicalité [i.e. la radicalité de la thèse de la phénoménologie de la donation] impliquerait non seulement que quelques phénomènes se déplient comme des phénomènes donnés et que quelques donnés s’ouvrent comme des phénomènes, mais que tout phénomène, en tant simplement qu'il apparaît, s'identifie toujours et d'emblée comme phénomène donné3 .

Richir et la phénoménologie cosmologique en tant que pensée de la phénoménalisation

Venons-en maintenant à la pensée de Marc Richir, qui constitue pour ainsi dire l'autre « pôle » de la nouvelle phénoménologie française. Notre étude se concentrera sur le jeune Richir et, plus précisément, sur ses « premiers écrits » dont la caractéristique et le motif ultime deviennent manifestes si nous les considérons avec Frédérique Streicher comme une "phénoménologie cosmologique » ${ }^{4}$. Comme nous le verrons, le jeune Richir caractérise lui-même son projet comme une « pensée de la phénoménalisation ».

\footnotetext{
${ }^{1}$ Voir aussi ibid., p. 159.

${ }^{2}$ Cette idée est bien sûr très critiquée, et pas seulement par Dominique Janicaud (qui n’a probablement pas saisi l'idée de Marion). Pour ne mentionner qu'un exemple de critique productive : voir Jocelyn Benoist, « Critique du donné », dans Archives de philosophie, numéro 73, 2010, p. 9-27).

${ }^{3}$ Jean-Luc Marion, Étant donné. Essai d'une phénoménologie de la donation (deuxième édition corrigée), Paris, PUF, 1998 (coll. «Épiméthée »), p. 247-248.

${ }^{4}$ Voir Frédérich Streicher, La phénoménologie cosmologique de Marc Richir et la question du sublime. Les premiers écrits (1970-1988), Paris, L’Harmattan, 2006.
} 
Il nous semble que l’œuvre de Richir se présente depuis ses premiers écrits — de manière beaucoup plus évidente que celle de Marion — comme une « réflexion sur (et une tentative de la transformation de) la phénoménalité du phénomène phénoménologique ». Richir déclare très explicitement tout au début de son premier ouvrage philosophique que le véritable point de départ de son projet (qui demeure toujours selon nous le moteur de sa pensée, malgré ses modifications radicales) est le suivant :

Elle [i.e. la doctrine husserlienne de la perception] est une expression achevée du cadre de la représentation, institué dans l'époque moderne depuis Descartes. Au lieu de reconstituer sa genèse historique, qui est extrêmement complexe et qui s'enracine dans la nouvelle réflexion sur l'espace effectuée à la Renaissance, nous en proposerons une analyse philosophique, pour en dégager in essentia toutes les implications ${ }^{1}$.

On remarquera ici que Richir considère la «doctrine husserlienne de la perception » (donc la théorie des Abschattungen) comme une forme de pensée qui renvoie nécessairement à une certaine institution historique, à savoir dans ce contexte à « la révolution cosmologique » de la Renaissance ${ }^{2}$. Si la «perception » telle que Husserl l'analyse n'est autre qu'une des «Sachen selbst » par excellence de la phénoménologie, il s'agit alors pour Richir de réfléchir et d'analyser "philosophiquement» l'origine et la genèse même du phénomène phénoménologique dans la pensée husserlienne. C'est pourquoi nous pouvons dire que Richir tente une reconduction philosophico-génétique de la phénoménalité avec laquelle la phénoménologie opère d'avance vers son origine . $^{3}$.

Comment s’accomplit cette recherche génétique ? Elle ne s'accomplit pour Richir que phénoménologiquement. Autrement dit, son projet n'est pas celui d'une histoire philosophique de la cosmologie (telle que l'entreprend par exemple Alexandre Koyré) ${ }^{4}$ :

\footnotetext{
${ }^{1}$ Marc Richir, Au-delà du renversement copernicien. La question de la phénoménologie et son fondement, Den Haag, Martinus Nijhoff (coll. « Phænomenologica »), 1976, p. 1. Nous soulignons. ${ }^{2}$ Ibid.

${ }^{3}$ Mais cette « origine » ne peut jamais être donnée selon Richir : «L’absolu n’est jamais présent, il n’y a pas d'origine assignable dans le registre de la phénoménologie, mais seulement un mythe de l'origine, et en ce sens, la phénoménologie pourrait devenir elle-même poétique de l'origine» (Marc Richir, Recherches phénoménologiques (I, II, III). Fondation pour la phénoménologie transcendantale, Bruxelles, Ousia, 1981, p. 25). Nous voyons ici une certaine "proximité » entre la pensée richirienne et la pensée finkienne : «Wenn ein Seiendes gegeben ist, so ist sein „Ursprung“ in keiner Weise mehr, auch nicht für Gott mehr originär gebbar [...] Ontische Ursprungsfrage ist Frage nach Vorhin, nach dem Woher. Gefragt ist ein innerzeitlich Vergangenes. Phänomenologische Ursprungsfrage ist Frage nach der Zeitlichkeit dieses Seienden » (Eugen Fink Gesamtausgabe, vol. 3/1, R. Bruzina (éd.), Freiburg/München, Alber, 2006, p. 219).

${ }^{4}$ Voir Alexandre Koyré, La révolution astronomique, Paris, Hermann, 1961, et Alexandre Koyré, Du monde clos à l'univers infini, Paris, Gallimard, 1973.
} 
Par phénoménologie, nous signifions la doctrine philosophique qui consiste à déployer et à rassembler dans le logos les rapports internes (logoi) que l'apparence (le phainomenon) suscite d'elle-même à partir d'elle-même, dans sa phénoménalisation et sa distorsion originaire [...] la phénoménologie [...] est le lieu d'une interrogation philosophique, qui, dès lors, se loge dans l'énigme de l'apparence et de sa phénoménalisation ${ }^{1}$.

C’est pourquoi Richir dit que sa tentative n'est rien autre qu'une «pensée de la phénoménalisation ». Cette pensée articule selon Richir «trois faits fondamentaux » : (1) "le fait qu'il y a apparence », (2) "le fait qu'il y a sensation de l'apparence » et (3) " le fait qu'il y a savoir du fait qu'il y a apparence, c'est-à-dire le fait que le savoir du fait n'est pas aveugle ou absolument positif, mais susceptible de s'interroger sur lui-même comme fait " ${ }^{2}$. Dans la mesure où elle cherche à élucider la phénoménalité du phénomène à partir du factum même d’apparaître en tant que tel, la pensée du jeune Richir n’est ni une déduction (métaphysico-spéculative) de ce fait à partir de quelque principe ou idéal transcendantal (car ce type de déduction s'accomplit, nous semble-t-il, nécessairement de manière spéculative), ni une simple description des faits empiriques (car il s'agit là de trois faits originaires que nous pouvons considérer avec Husserl comme des Ur-Fakta qui précèdent tout fait empirique et le rendent possible $)^{3}$.

Il nous faut poser dans ce contexte la question suivante : est-ce que l' "événement » détermine la pensée richirienne de la phénoménalisation ? Si oui, quel concept d'événementialité est à l'œuvre chez Richir ? De prime abord, l'événement de type historique nous semble constituer un modèle pour sa réflexion. Si la tâche de la pensée de la phénoménalisation consiste dans une reconduction génétique de la phénoménalité du phénomène phénoménologique (dont un exemple est la perception telle que Husserl l'analyse) à son origine, il est en effet nécessaire d'interroger (de manière purement philosophique) l'origine historique de la philosophie elle-même. « La philosophie est — dit Richir — historique, précisément parce qu'elle ne sait pas ce qu'elle est. Elle est rapport à l'Histoire parce que, par toute une part d'elle-

\footnotetext{
${ }^{1}$ Marc Richir, Au-delà du renversement copernicien, op.cit., p. 78-79.

${ }^{2}$ Ibid.

${ }^{3}$ En ce qui concerne l' « Ur-faktum » chez Husserl, voir en particulier le texte 22 de Hua, vol. XV (Edmund Husserl, Zur Phänomenologie der Intersubjektivität. Texte aus dem Nachlass. Dritter Teil. 1929-35, I. Kern (éd.), Den Haag, Martinus Nijhoff, 1973). Pour une analyse approfondie de ce texte, voir Marc Richir, Méditations phénoménologiques, Grenoble, Jérôme Million, 1992, p. 380-389. Pour une présentation générale de cette problématique à la lumière de l'égologie husserlienne, voir Stefano Micali, Überschuß der Erfahrung Grenzdimensionen des Ich nach Husserl, Dordrecht, Springer, 2008, p. 79-100. Pour une approche approfondie du problème dans la perspective de la tradition métaphysique, voir László Tengelyi, Welt und Unendlichkeit, op. cit., p. 171191. Pour une continuation de cette problématique dans la phénoménologie japonaise contemporaine, voir enfin notre étude, « Das Konzept der Phänomenologie der transzendentalen Medialität bei Yoshihiro Nitta "Faktizität und ihre transzendental-mediale Funktion” ", dans Interpretationes, numéro 5, 2015 (à paraître).
} 
même qui la retourne sur elle-même dans l'interrogation sans fin de son origine, elle est prise par la dimension barbare de l'Histoire, et elle la réfléchit » ${ }^{1}$. L’Histoire a nécessairement sa « dimension barbare » parce que pour Richir : «L'Histoire est un avènement qui dépasse toujours l'événement ; un avènement toujours imminent, de l'imminence même de la sauvagerie qui fait l'Histoire »" Autrement dit, Richir entend par Histoire l'« avènement» ou l'"événement» qui rend possible ce que nous appelons des "événements normalisés » (c'est pourquoi un «avènement dépasse toujours l'événement »). Ainsi, l'Histoire ne s'avère en tant qu'Histoire que dans une expérience «barbare », sans précédent. L’Histoire a nécessairement pour la philosophie (ou pour l'activité de philosopher elle-même) une dimension de sauvagerie, parce que son avènement (ce à partir de quoi le phénomène s'avère) ne peut être reconnu en tant qu'avènement que si (selon l'expression de Marion) il « interloque » le philosophe. Deuxièmement, il faut prendre au sérieux le fait que Richir entreprend dans son ouvrage une " analyse philosophique » de la cosmologie et plus précisément de la doctrine spéculative de l'espace de Nicolas de Cues jusqu'au dernier Merleau-Ponty. Le «cosmos » de la cosmologie ou l'espace n'est rien d'autre que la dimension $d u$ «monde » dans lequel tout événement arrive. Parce que l'enjeu de la pensée richirienne consiste dans l'analyse de la phénoménalisation même du « monde » dans lequel tout phénomène se révèle, nous pouvons la comprendre comme un approfondissement de la thèse finkienne du «monde » en tant qu'événementiel (même si Richir ne la connaissait sans doute pas à l'époque de la rédaction d'Audelà du renversement copernicien).

Nous pouvons ainsi répondre à la question de savoir quelle polarisation est en jeu dans la nouvelle phénoménologie française : d'une part, Richir met en œuvre une "pensée de la phénoménalisation » dont la tâche consiste à élucider de manière génétique la phénoménalité et dont l'accomplissement transforme le concept de phénomène; d'autre part, Marion met en œuvre, comme nous venons de le montrer, une radicalisation de la réduction phénoménologique qui transforme le concept de phénomène.

\section{B/ Les contributions de la nouvelle phénoménologie à la tradition métaphy- sique \\ Marion et la lutte contre l'onto-protologie}

Parce que Marion affirme, comme nous l'avons vu, que la troisième " percée » de la phénoménologie de la donation consiste dans une réflexion radicale sur tout type de phénomène à partir de sa donation (dans nos propres termes, à partir de son « événementialité »), il met phénoménologiquement à l'épreuve dans Étant donné «quelques catégories de l'étant, qui se redéfinissent radicalement une fois appli-

\footnotetext{
${ }^{1}$ Marc Richir, Au-delà du renversement copernicien, op. cit., p. 130.

${ }^{2}$ Ibid., p. 131.
} 
quées au phénomène donné : ainsi contingence et nécessité (§14-15), accident et substance (§16), cause et effet (§17) s’inversent et se recomposent à partir de la donation $"$. Bref, la tâche ultime de la phénoménologie de la donation culmine dans une révision des catégories fondamentales de la métaphysique depuis Aristote. Nous ne pourrons examiner ici que très brièvement la révision de la catégorie de " contingence ", qui selon Neue Phänomenologie in Frankreich détermine l'orientation de Étant donné. Elle atteste très clairement la thèse même de nos auteurs :

Avant de dire le simple contraire du nécessaire, le contingent dit ce qui me touche, ce qui m'atteint et donc ainsi m'arrive (selon le latin), ou (selon l'allemand [Zufälliges]) ce qui « tombe comme ça », donc «me tombe dessus ». Le phénomène apparaît dans la mesure où d'abord il vient, pousse et s'étend jusqu'à moi (il me devient contigu, il entre en contact avec moi), pour, ensuite, m'affecter (agir sur moi, me modifier). Aucun phénomène ne peut apparaître sans m’advenir, m’arriver, m'affecter à titre d'événement, qui modifie mon champ (de vision, de connaissance, de vie, peu importe ici) ${ }^{2}$.

En partant de cette thèse, que nous partageons entièrement avec Marion, Étant donné essaye de résoudre ce qu'il appelle les « apories du sujet », que nous ne pourrons étudier ici $^{3}$. Il nous faut néanmoins souligner le fait que la dissolution de ces apories doit toujours renvoyer, selon Marion, à une analyse phénoménologique de ce que nous appelons « sujet au datif » ${ }^{4}$. Par précaution, nous rapporterons les termes mêmes de Marion :

Il ne s'agit plus de se comprendre au cas nominatif (visant l'objet : Husserl), ni au génitif (de l'être : Heidegger), ni même selon l'accusatif (accusé par autrui — Levinas), mais selon le datif : je me reçois de l'appel qui me donne à moi-même, avant de me donner quoi que ce soit. Il faudrait presque supposer que cet étrange datif ne se distingue plus ici de l'ablatif, puisque le moi/me rend possible (comme ouvrier, moyen), en tant que premier don départi par l'appel, l'ouverture de toutes autres donations de donnés particuliers ${ }^{5}$.

C’est pourquoi la thèse de Marion est beaucoup moins douteuse qu'elle n’y paraît de prime abord : Marion caractérise le sujet au datif comme «l'adonné » par excellence, parce qu’il s’est adonné nécessairement à l’ouverture même de données ${ }^{6}$.

\footnotetext{
${ }^{1}$ Jean-Luc Marion, Étant donné, op.cit., p. 249.

2 Ibid., p.177. Voir aussi Hans-Dieter Gondek et László Tengelyi, Neue Phänomenologie in Frankreich, op.cit., p. 229-238.

${ }^{3}$ Jean-Luc Marion, Étant donné, op.cit., p. 343-373. Voir aussi Hans-Dieter Gondek et László Tengelyi, Neue Phänomenologie in Frankreich, op.cit., p. 191-195.

${ }^{4}$ Jean-Luc Marion, Étant donné, op.cit., p. 369-373. Voir aussi Hans-Dieter Gondek et László Tengelyi, Neue Phänomenologie in Frankreich, op.cit., p. 194.

${ }^{5}$ Jean-Luc Marion, Étant donné, op.cit., p. 371.

${ }^{6}$ Ibid., p. 369-373. En ce qui concerne «l'adonné » chez Marion, nous avons deux remarques qui le mettent en rapport avec la phénoménologie d'Eugen Fink. Premièrement, Fink analyse déjà dans "Vergegenwärtigung und Bild » (paru en 1930) les différents modes de la structure du "versunken in » (l'«absorbé dans », selon la traduction de Didier Franck), ou de la «Versunkenheit » («ab-
} 
Cette transformation de la phénoménalité du phénomène, qui s’accompagne de celle du sujet, conduit ainsi Marion à une révision foncière des catégories métaphysiques, qui à la fois suit et critique Heidegger. Autrement dit, nous pouvons sans doute caractériser le projet de Marion comme un approfondissement de la lutte heideggérienne contre la tradition métaphysique que nous appelons l'onto-protologie, dont on croit pouvoir trouver l'expression la plus achevée chez Leibniz, précisément dans sa formulation du principium rationis (der Satz vom Grund). Cet approfondissement s'accomplit néanmoins de manière très critique vis-à-vis de la pensée heideggerienne, dans la mesure où Marion, loin de répéter simplement la critique de la « métaphysique de la subjectivité », réhabilite l'analyse et la réduction phénoménologique, comme reconduction à l'expérience du sujet au datif.

\section{Richir et une réhabilitation de la tradition cosmo-hénologique}

Parce que la pensée de la phénoménalisation s'accomplit chez le jeune Richir comme une phénoménologie de la cosmologie, sa tâche consiste à élucider le logos même du monde ou bien sa phénoménalisation, à partir de laquelle le monde « est ». Comme on le sait, la cosmologie et l'astronomie ont été transformées une fois pour toutes dans notre histoire par ce qu'on appelle habituellement le «renversement

sorption ») en tant qu'accès originaire de la conscience (voir Eugen Fink, « Vergegenwärtigung und Bild. Beiträge zur Phänomenologie der Unwirklichkeit » (1930), in Studien zur Phänomenologie 1930-1939, Den Haag, Martinus Nijhoff, 1966, p. 55 sq). Cette terminologie finkienne ressemble remarquablement à « l'adonné » chez Marion. Dans la mesure où la terminologie de «Versunkenheit " n'a aucune connotation religieuse chez Fink (il ne s'agit jamais de "Versunkenheit » dans le sens de la méditation ou de la pratique religieuse), nous pourrions même la préférer dans certains contextes à «l'adonné ». Deuxièmement, ce n'est pas un hasard que ces deux phénoménologues aient forgé ces concepts dans le cadre d'une analyse phénoménologique de l'image. La différence entre les deux est également manifeste. Alors que Marion s'occupe depuis toujours des analyses phénoménologiques de l'idole et de l'icône (non seulement dans Étant donné, mais aussi dans L'idole et la distance et dans Dieu sans l'être), Fink entend le phénomène d'image (Bildphänomen) non seulement comme peinture ou photographie (dont Husserl analysa soigneusement la constitution dans ses cours et manuscrits (voir Edmund Husserl, Phäntasie, Bildbewusstsein, Erinnerung. Zur Phänomenologie der anschaulichen Vergegenwartigungen. Texte aus dem Nachlass (1898-1925), dans Hua, vol. XXIII, E. Marbach (éd.), Den Haag, Martinus Nijhoff, 1980)), mais aussi explicitement comme «jeu » (Spiel) (voir Eugen Fink, «Vergegenwärtigung und Bild», dans Studien zur Phänomenologie, op. cit., p. 71). Cette conception du jeune Fink culminera plus tard dans sa fameuse analyse du jeu en tant que « reflet » (Widerschein) du monde. Voir Eugen Fink, Spiel als Weltsymbol, Stuttgart, Kohihammer, 1960. Voir aussi l'avertissement pour la traduction française de Studien zur Phänomenologie 1930-1939: Eugen Fink, De la phénoménologie, tr. fr. par D. Franck, Les Éditions de minuit, 1974, p. 8) et l'excellente analyse de László Tengelyi, "Finks Fenster ins Absolute", dans Phänomenologische Forschungen, vol. XXX, E.W. Orth (éd.), Freiburg/München, Alber, 1996 (dont il y a aussi une version française : «La "fenêtre sur l'absolu" selon Fink », dans Eugen Fink. Actes du Colloque de Cerisy-la-Salle, 23-30 juillet 1994, N. Depraz et M. Richir (éds.), Amsterdam/Atlanta, Rodopi, 1998). 
copernicien $" 1$. L'expression philosophico-spéculative de ce renversement remonte premièrement à Nicolas de Cues et à Giordano Bruno.

Bien que les implications et la genèse de l'expression philosophique du « renversement copernicien » soient extrêmement complexes et que Richir le sache bien, sa stratégie consiste très clairement à interpréter et analyser le pli de ce «renversement " à partir de ce qui nous rend intelligible le monde en tant que cosmos «renversé » ou à partir de l'«ouverture » même où nous le reconnaissons en tant que cosmos «renversé » ${ }^{2}$. Plus précisément, il s'agit pour Richir du problème de la « vision » (il développe ici la pensée du dernier Merleau-Ponty) : son analyse se propose de rendre intelligible tel lieu à partir duquel le monde est « $v u »$. Sa thèse peut être présentée de la manière suivante. Préalablement au "renversement », la cosmologie est strictement liée à une position du « survol », que l’on peut retrouver (avec de

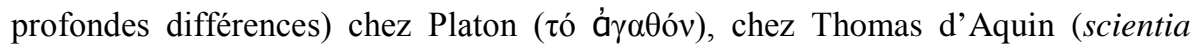
Dei), etc. : le cosmos est ainsi interprété comme monde fini à partir de l'infini, en tant que « centre absolu » du survol (par exemple de Dieu). La cosmologie instituée à la suite du « renversement copernicien » (que Richir appelle " cosmologie classique ») présuppose en revanche explicitement une « métaphysique de la finitude » ${ }^{3}$, qui institue « une représentation de l'infini à partir du fini et dans laquelle le fini ne cesse de hanter l'infini " ${ }^{4}$. Cette «métaphysique de la finitude » (telle que Richir l'explicite dans $A u$-delà du renversement copernicien) n’est saisissable qu'à la lumière d'une proposition issue du corpus hermeticum : le monde est «la sphère infinie dont le centre est partout et la périphérie nulle part ${ }^{5}$. Comme le montre selon Richir la théorie husserlienne de l'intersubjectivité, mon point de vue ou encore la vision qui m’appartient en tant qu'ego n’a pas de valeur privilégiée dans le cadre de la « constitution du monde objectif », dans la mesure où un sujet fini doit nécessairement coexister avec d'autres points de vue (ceux des autres ego) pour pouvoir constituer le monde en tant que système spatio-temporel objectif ${ }^{6}$. Le monde est

\footnotetext{
${ }^{1}$ L'expression « renversement copernicien » est plutôt mal choisie dans la mesure où « Copernic conserve la cosmologie ancienne puisqu'il garde l'idée d'un centre absolu de l'univers ». Voir Marc Richir, Au-delà du renversement copernicien, op. cit., p. 27, où il renvoie aux travaux déjà cités de Koyré et surtout de Serres.

${ }^{2}$ Voir Frédérich Streicher, La phénoménologie cosmologique de Marc Richir et la question du sublime, op. cit., p. 46.

${ }^{3}$ Marc Richir, Au-delà du renversement copernicien, op. cit., p. 24.

${ }^{4}$ Ibid., p. 21.

${ }^{5}$ " Deus est sphaera infinita cuius centrum est ubique, circumferentia vero nusquam » (Liber XXIV philosophorum, prop. 2, tr. fr. par F. Hudry, Le livre des XXIV philosophes, Grenoble, Jérôme Million, 1989, p. 95).

${ }^{6}$ Voir Marc Richir, Au-delà du renversement copernicien, op. cit., p. 27-29 et Frédérich Streicher, La phénoménologie cosmologique de Marc Richir et la question du sublime, op. cit., p. 81-125. Surgit ici — notamment pour le dernier Husserl — le problème du "Ur-Ich », qui peut sembler de prime abord (du moins pour non-spécialiste) un «centre absolu » par rapport auquel l'autrui ne serait que la « périphérie » (voir Edmund Husserl, Die Krisis der europäischen Wissenschaften und
} 
alors interprété, selon l'expression philosophique achevée du « renversement copernicien ", comme étant la sphère infinie que le sujet fini constitue infiniment avec d'autres sujets finis : il s'agit d'un « fini infinitisé » ${ }^{1}$. Le mouvement constitutif «infinitisé » d'un sujet fini avec les autres sujets finis s'oriente ainsi, comme le dit Richir, "téléologiquement», sans jamais parvenir à une constitution complète du monde. Mais vers quoi s'oriente-t-il ? L'orientation de toutes les orientations de ce mouvement téléologique n'est autre que «l'Idée » du monde objectif : une «idée régulatrice » au sens kantien ${ }^{2}$. Or, Richir interprète (en se référant à la doctrine des «mondes possibles » de Leibniz) la nature même de l'idée cosmologique (donc de l'idéalité même de l'idée cosmologique) de la manière suivante : "Chaque Idée est origine de l'ensemble et point de vue sur l'ensemble à partir de l'origine »". L'idée régulatrice au sens kantien est la régulation originairement unifiante de l'ensemble, autrement dit, le monde est ici l'idée régulatrice de l'ensemble des choses mondaines et il s'agit là du point de vue à partir duquel le monde s'avère tel qu'il est interprété dans le cadre cosmologique du «renversement copernicien ». Pour accéder à la genèse des implications philosophiques mêmes du « renversement copernicien ", Richir fait dans Le Rien et son apparence une importante digression à travers le «mythe de la caverne » (il critique la lecture heideggérienne du texte platonicien en développant la pensée du dernier Merleau-Ponty sur le «narcissisme » de la vision $)^{4}$ et, en partant de l'institution platonicienne de la philosophie dont la difficulté suscite l'institution néoplatonicienne de la philosophie et ce qu'il appelle le " néo-platonisme copernicien » (surtout Nicolas de Cues et G. Bruno), il croit trouver le « fondement » spéculatif de leur dépassement dans l'idéalisme allemand (surtout chez le jeune Fichte) $)^{5}$. Le dépassement du «renversement copernicien » est

die transzendentale Phänomenologie. Eine Einleitung in die phänomenologische Philosophie, dans Hua, vol. VI, W. Biemel (éd.), Den Haag, Martinus Nijhoff, 1976). Il semble dès lors qu'il y ait une hiérarchie absolue orientée à partir de l' «Ur-Ich » (comme " centre absolu » ou comme position de survol). La doctrine husserlienne de l' «Ur-Ich » n'implique au contraire (comme le montre Shigeru Taguchi de manière convaincante) aucune connotation ou aucun retour à l'interprétation quasi onto-théologique d'une quelconque causa prima. Voir Shigeru Taguchi, Das Problem des "Ur-Ich" bei Edmund Husserl. Die Frage nach der selbstverständlichen "Nähe" des Selbst, Dordrecht, Springer, 2006.

${ }^{1}$ Marc Richir, Au-delà du renversement copernicien, op. cit., p. 22, 83.

${ }^{2}$ Ibid., p. 28.

${ }^{3}$ Ibid., p. 10-11.

${ }^{4}$ « De sorte que le voyant étant pris dans cela qu'il voit, c'est encore lui-même qu'il voit : il y a un narcissisme fondamental de toute vision ; et que, pour la même raison, la vision qu'il exerce, il la subit aussi de la part des choses, que, comme l'ont dit beaucoup de peintres, je me sens regardé par les choses, que mon activité est identiquement passivité - ce qui est le sens second et plus profond du narcissisme » (Maurice Merleau-Ponty, Le visible et l'invisible, Paris, Gallimard, 1964, p. 181).

${ }^{5}$ Marc Richir, Le Rien et son apparence. Fondements pour la phénoménologie (Fichte: Doctrine de la Science 1794/1795), Bruxelles, Ousia, 1979. Voir Frédérich Streicher, La phénoménologie cosmologique de Marc Richir et la question du sublime, op. cit., p. 29-77. 
selon Richir philosophiquement indispensable, parce que ce dernier ne peut rendre intelligible ce que Merleau-Ponty appelle « la diplopie ontologique » ${ }^{1}$. Il s'agit pour Richir de la difficulté à rendre compte de la genèse de la dualité entre « le fini » et «l'infini $»^{2}$. Le jeune Fichte (avec sa théorie de l'Anstoss, du « choc ») ${ }^{3}$ et le dernier Merleau-Ponty (avec sa philosophie de la chair) étaient en train d'achever le dépassement du «renversement copernicien»; ainsi leurs philosophies ne sont rien d'autre que le mouvement vers son « au-delà ». Aux yeux de Richir, les deux philosophes tentent en effet tous deux de trouver le " fondement » philosophique spéculatif d’une pensée de la phénoménalisation. Selon l’interprétation richirienne, « l'Anstoss » n'est rien d'autre que le « fait » à partir duquel s'articulent les « trois faits fondamentaux » de la phénoménalisation, qui est en soi le «rien ». Quant à Merleau-Ponty, la conception de la « chair » n'a pas d'instance transcendante à ellemême : il n'y a que la «chair », donc pas de contre-chair ou d'au-delà. Elle est l'élément de l' «Infini » : l' «infini d'Offenheit et non pas Unendlichkeit — Infini du Lebenswelt et non pas infini d'idéalisation — Infini négatif, donc — Sens ou raison qui sont contingence » ${ }^{4}$. Nous pouvons d’ores et déjà conclure que la pensée de la phénoménalisation chez Richir ${ }^{5}$ réhabilite et prolonge (sans le savoir) la conception phénoménologique finkienne $d u$ "monde » en tant qu'événementiel : le monde se phénoménalise en effet pour lui à partir de "trois faits fondamentaux » et ces trois faits ne sont que le pli de la contingence fondatrice ou l'événement par excellence pour tout phénomène. Ces trois faits sont contingents, parce qu'ils nous arrivent originairement, mais aussi fondateurs ou "possibilisants », parce qu'il n'y aurait aucun phénomène sans le fait même de l'apparaître. On parvient ainsi à comprendre plus précisément en quoi consiste pour nous la différence entre les « deux pôles extrêmes » de la nouvelle phénoménologie française : alors que Marion approfondit

\footnotetext{
1 «Le caractère essentiel de la diplopie est de se trouver lié à une dualité (le sensible et l'intelligible, le fini et l'infini, etc...), irréductible par l'anéantissement de l'un des termes dans l'autre, dans la mesure où elle est reportée à un autre niveau (l'illusion pure et la vérité pure, la transparence pure et l'opacité pure), de telle sorte que, comme l'entendait Merleau-Ponty, il ne reste d'autre possibilité que d'en "prendre possession entière, comme le regard prend possession des images monoculaires pour en faire une seule vision” " (Marc Richir, Le Rien et son apparence. Fondements pour la phénoménologie (Fichte : Doctrine de la Science 1794/1795), op. cit., p. 20. Voir aussi Maurice Merleau-Ponty, Résumés de cours. Collège de France 1952-1960, Paris, Gallimard, 1968, p. 125-128).

${ }^{2}$ Ibid.

${ }^{3}$ Car Richir interprète le choc comme ce à partir de quoi le rapport entre moi et non-moi se phénoménalise. Voir en particulier Marc Richir, Le Rien et son apparence, op. cit., p. 307-308.

${ }^{4}$ Maurice Merleau-Ponty, Le visible et l'invisible, Paris, Gallimard, 1964, p. 221.

${ }^{5}$ Richir met au point dans les Recherches phénoménologiques (Bruxelles, Ousia, 1981-1983) et dans les Méditations phénoménologiques (Grenoble, Jérôme Millon, 1992) la fondation de la méthode de sa "phénoménologie du langage » (la « réduction hyperbolique »), et met en œuvre des analyses génétiques concrètes de l'institution de la langue philosophique et mythologique - en abordant Kant et Schelling — dans L'expérience du penser (Grenoble, Jérôme Million, 1996).
} 
la lutte heideggérienne contre la tradition « onto-protologique » de la métaphysique en renversant la pensée de Heidegger, Richir réhabilite la tradition «cosmohénologique » de la métaphysique en approfondissant la pensée de Fink et du dernier Merleau-Ponty ${ }^{1}$.

\section{Conclusion}

Ainsi se trouve atteint et mis au jour un aspect qui permet de relativiser la thèse de Janicaud selon laquelle la nouvelle phénoménologie en France confondrait phénoménologie et théologie. Comme on l'a montré en suivant quelques-unes des thèses de Neue Phänomenologie in Frankreich, il ne s'agit pas en effet d'une reconduction des phénomènes à l'Avènement par excellence, mais plutôt d'une réflexion philosophico-phénoménologique sur le phénomène dans son événementialité (le phénomène en tant qu'événement de sens). Cela signifie aussi que la nouvelle phénoménologie française ne se limite pas à une compréhension classique de la phénoménalité à partir de sa constitution dans la subjectivité transcendantale. La spécificité de la nouvelle phénoménologie s’avère consister plutôt dans le fait de déployer l’intuition philosophique suivante : analyser le phénomène non pas à partir d'un " possible » qui précéderait le « réel » (ce qu’on pourrait caractériser lato sensu comme une sorte de platonisme), mais analyser le phénomène à partir d'un « réel » qui rend possible le « possible ». Cette «possibilisation » (Ermöglichung) ne doit pas être confondue avec un rapport de conditionnement d'une instance par une autre qui serait d'une nature étrangère à la première, parce qu'il ne s'agirait là (du moins pour la phénoménologie) que d'une Metabasis ${ }^{2}$. Il s'agit en revanche de comprendre la possibili-

\footnotetext{
${ }^{1}$ Richir commence à aborder la pensée finkienne après la parution de la sixième méditation cartésienne (Eugen Fink, VI. Cartesianische Meditation. Texte aus dem Nachlass Eugen Finks (1932) mit Anmerkungen und Beilagen aus dem Nachlass Edmund Husserls (1933/34), Dordrecht/Boston/New York/London, Kluwer Academic Publisher, 1988), des recherches sur ses inédits (voir les travaux de Ronaldo Bruzina) et de ses cours inédits (comme par exemple, Welt und Endlichkeit, paru en 1990). Il prend dès lors l'initiative de donner à la recherche finkienne un espace francophone : voir Marc Richir, « La question d'une doctrine transcendantale de la méthode en phénoménologie », dans Épokhè, numéro 1, 1990, p. 91-125 ; Marc Richir, « Mondes et phénomènes », dans Les Cahiers de Philosophie, numéros 15-16, 1993, p. 71-88 ; Marc Richir, « Temps, espace et monde chez le jeune Fink », dans Eugen Fink. Actes du Colloque de Cerisy-la-Salle, op. cit., p. 27-42. Pour une Auseinandersetzung approfondie entre Richir et la pensée finkienne, voir Marc Richir, Méditations phénoménologiques, op. cit., p. 338-362 ; Marc Richir, « Schwingung et phénoménalisation - Heidegger, Fink, Husserl, Patočka », dans Internationale Zeitschrift für Philosophie, numéro 1, 1998, p. 52-63.

2 « Die fehlerhafte Metabasis in psychologistische und metaphysische Gedankengänge bringt die Unklarheit ; sie schafft die Scheinprobleme und die Scheintheorien zu ihrer Lösung " (Edmund Husserl, Logische Untersuchungen. Zweiter Teil. Untersuchungen zur Phänomenologie und Theorie der Erkenntnis, dans Hua, vol. XIX/1, U. Panzer (éd.), Den Haag, Martinus Nijhoff, 1984, p. 154).
} 
sation ou la phénoménalisation du phénomène à partir d'elle-même. Mais cette auto-

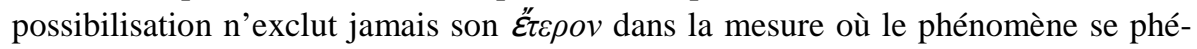
noménalisant articule deux dimensions en les possibilisant : le phénomène possibilisant et le phénomène possibilisé (par exemple la Révolution française et la vie du citoyen moderne), le phénomène caché et se manifestant (par exemple l'être et l'étant chez Heidegger, l'énigme du Sphinx et l'homme chez les grecs), etc. Si nous pouvons parler effectivement de cette auto-possibilisation différenciante du phénomène, ce n'est que dans la mesure où nous ne l'entendons rigoureusement qu'à partir du caractère événementiel de son avènement. Un événement surprenant engendre en effet la dimension même de l'ouverture originaire où il peut nous arriver, autrement il arriverait sans nous interloquer, il ne serait que le cas particulier d'une règle générale. C’est pourquoi nous avons insisté sans cesse sur le fait qu’il n’est pas question dans la nouvelle phénoménologie française d'une spécification descriptive d'événements particuliers, mais de l'accomplissement d'une réflexion philosophique sur la phénoménalité phénoménologique dans son événementialité. Il faut admettre néanmoins que cette tentative risque dans certains contextes de tomber dans une Metabasis phantasmatique, on pourrait dire dans une illusion transcendantale phénoménologique, à savoir celle de fonder des phénomènes empiriques sur une forme spécifique d'événement. En tout cas, l'orientation originaire de la nouvelle phénoménologie française ne consiste pas simplement dans une réflexion philosophique sur le phénomène compris dans son événementialité, mais aussi dans une critique de toute fondation des phénomènes sur un événement par excellence (comme le Vendredi saint semble l'être pour Hegel) ${ }^{1}$. La spécificité de l'attitude de la « nouvelle phénoménologie française » par rapport à la "phénoménologie française » s’avère dès lors consister dans le fait de ne pas se réduire à l'analyse d'une forme spécifique de phénomène événementiel, mais d'accomplir une réflexion philosophique générale sur le phénomène dans sa dimension événementielle, alors que la génération précédente s'occupa plutôt d' « hyper-phénomènes » ou de « contre-phénomènes »².

\footnotetext{
${ }^{1}$ Hegel parle de «speculativen Charfreytag » : voir Georg Wilhelm Friedrich Hegel, «Glauben und Wissen », in Gesammelte Werke, vol. IV : Jenaer kritische Schriften, H. Buchner et O. Pöggeler (éds.), Hamburg, Meiner, 1968, p. 414.

2 Tels que la peinture chez Merleau-Ponty, le « visage » chez Levinas, le symbole chez Ricœur, l'affectivité de la Vie chez Henry, etc. Voir Hans-Dieter Gondek et László Tengelyi, Neue Phänomenologie in Frankreich, op.cit., p. 24-25.
} 